Keith L. Williams • Eric D. Pakarinen

James H. Woods

\title{
Quadazocine decreases responding reinforced by ethanol, sucrose, and phencyclidine fluid deliveries in rhesus monkeys: comparison to naltrexone's effects
}

Received: 24 June 1998 / Accepted: 18 February 1999

\begin{abstract}
Rationale: The endogenous opioid system may mediate the reinforcing effects of ethanol as well as sweet-tasting solutions. For example, opioid antagonists, such as naltrexone, reduce ethanol- and sucrose-reinforced responding in rhesus monkeys. If these effects are due to blockade of the $\mu$-receptor, then an opioid antagonist such as quadazocine with a receptor selectivity profile similar to that of naltrexone should reduce responding at doses correlated with its $\mu$-selectivity. Objectives: To determine whether quadazocine would reduce responding for ethanol and sucrose at $\mu$-selective doses, and whether quadazocine and naltrexone would reduce responding for a bitter-tasting drug solution such as phencyclidine. Methods: Rhesus monkeys were given access to ethanol, sucrose, or phencyclidine concurrently with water. Prior to the drinking sessions, quadazocine $(0.032-3.2 \mathrm{mg} / \mathrm{kg})$ or saline was injected intramuscularly. During the phencyclidine experiment, naltrexone $(0.1$ and $0.32 \mathrm{mg} / \mathrm{kg}$ ) was also tested. Results: The highest quadazocine doses (1 and $3.2 \mathrm{mg} / \mathrm{kg}$ ) reduced ethanol and sucrose fluid deliveries without affecting the concurrently available water. Quadazocine reduced the fluid deliveries of both phencyclidine and water when concurrently available. Naltrexone reduced only phencyclidine fluid deliveries. Conclusions: The opioid antagonist effect on oral-reinforced responding is not selective for ethanol or sweet-tasting solutions; responding for phencyclidine was reduced as well. Quadazocine and NTX may reduce responding by blocking the $\mu$-receptor because the relative potency of these antagonists to reduce oral self-administration was similar to their relative potency to produce withdrawal in morphine-dependent monkeys. However, water responding was low in these
\end{abstract}

K.L. Williams · J.H. Woods

Department of Psychology, University of Michigan,

Ann Arbor, MI 48109-0632, USA

K.L. Williams $(\bullet)$ E.D. Pakarinen · J.H. Woods

Department of Pharmacology, 1301 MSRB III,

University of Michigan, Ann Arbor, MI 48109-0632, USA

e-mail: keithwil@umich.edu

Fax: +1-734-764-7118 experiments, and thus we cannot rule out rate-dependent effects of the antagonists.

Key words Opioid antagonists - Alcohol reinforcement . Self-administration $\cdot$ Primates

\section{Introduction}

The endogenous opioid system modulates alcohol drinking. In preclinical and clinical studies, opioid antagonists reduce alcohol drinking. For instance, we have previously shown that naltrexone (NTX) pretreatment in rhesus monkeys reduced oral ethanol self-administration (Williams et al. 1998). In other studies using many different animal species, NTX and other opioid antagonists reduced oral ethanol self-administration (Levine and Billington 1989). When ethanol was available concurrently with water, the opioid antagonist naloxone selectively reduced ethanol drinking (De Witte 1984; Froehlich et al. 1990). Because opioid antagonists effectively reduced ethanol drinking in animals, clinical trials tested the longer lasting opioid antagonists NTX and nalmefene, in alcohol-dependent patients. NTX and nalmefene increased abstinence, decreased relapse rates, and reduced alcohol craving (O'Malley et al.1992; Volpicelli et al. 1992; Mason et al. 1994).

The endogenous opioid system may mediate ingestive behaviors in general rather than specifically ethanol-related behaviors (Levine et al. 1985). Although effective in the clinical trials with alcoholics, opioid antagonists reduce the consumption of many solutions and foods. For example, naloxone pretreatment in rats reduced feeding as well as water and sucrose drinking (Holtzman 1974; Maickel et al. 1977; Stapleton et al. 1979). Thus, opioid antagonists may mediate reductions in alcohol drinking through a mechanism that modulates a wide class of stimuli that support food or fluid consumption.

The ability of opioid antagonists to reduce consumption depends upon activity at opioid receptors. The antagonist effects are stereoselective; in some experiments, 
the inactive naloxone stereoisomer had no effect on consumption (Brown and Holtzman 1980; Kirkham and Cooper 1988). Furthermore, the rank order of the capacity of different antagonists to suppress water drinking corresponded with the rank order of antagonist potency to precipitate morphine withdrawal (Brown and Holtzman 1980). Therefore, the antagonist's interaction with the $\mu$-opioid receptors may mediate the reduction in consumption.

The opioid antagonists NTX and quadazocine have been well characterized, in vivo and in vitro. For example, NTX blocked the analgesic effect of the $\mu$-agonist alfentanil with greater potency than it blocked the analgesic effect of the $\mathrm{K}$-agonists U69,593 and bremazocine (Ko et al. 1998). In monkeys for whom responding was reinforced by food delivery, the opioid antagonist quadazocine blocked the rate-decreasing effects of the $\mu$-agonists alfentanil and fentanyl with greater potency than it blocked the rate-decreasing effects of the $\kappa$-agonists U69,593 and EKC. Thus, in vivo, NTX and quadazocine more potently block $\mu$-receptors than $\kappa$-receptors. An in vitro experiment using monkey brain showed that both NTX and quadazocine had greatest affinity for the $\mu$-receptor, then $\kappa$ - and $\delta$-receptors (Emmerson et al. 1994). Even though the receptor affinity order is similar for NTX and quadazocine, higher quadazocine doses are required to produce the same effects as NTX. For instance, a 10-fold higher quadazocine dose was needed to precipitate withdrawal and generalize to an NTX stimulus in morphine-dependent monkeys (Valentino et al. 1983; France et al. 1990). Because morphine produces its effects through the $\mu$-receptor, we can predict that if NTX reduces oral-reinforced responding through $\mu$-receptor antagonism, then quadazocine should produce similar effects at 10 -fold higher doses.

The purpose of this study was to determine if quadazocine reduced ethanol- and sucrose-reinforced responding and to determine if both quadazocine and NTX reduced responding reinforced by fluid deliveries of the NMDA antagonist phencyclidine (PCP). Quadazocine was tested on ethanol-reinforced responding because some research suggests that endogenous opioid activity mediates ethanol's reinforcing effects and that opioid antagonists reduce ethanol consumption by blocking ethanol-induced opioid activity (Ulm et al. 1995). Quadazocine was tested on sucrose-reinforced responding because sucrose is a non-drug reinforcer that maintains a large amount of responding. Quadazocine and NTX were tested on responding reinforced by PCP deliveries because PCP has quinine-like taste properties (Aspen 1997) and, while orally reinforcing (Carroll 1982), it lacks calories. We compared the quadazocine and NTX doses required to reduce oral-reinforced responding in order to ascertain whether NTX reduces oral-reinforced responding through an opioid mechanism. In addition, a choice procedure was used because it eliminates the need for additional days or groups to control for solution access and it provides a means to determine the reinforcing effect of the test solution.

\section{Materials and methods}

Experiment 1: ethanol self-administration

Subjects

Subjects were adult rhesus monkeys (Macaca mulatta; weighing $5.8-10.7 \mathrm{~kg}$ ) maintained at approximately $80 \%$ of their free-feeding weights. For experiment 1, two of the five male subjects previously participated in a study where they received NTX prior to ethanol access. In all of the following experiments the "Guide for the Care and Use of Laboratory Animals" (NIH publication, vol. 25, number 28, revised 1996) was followed.

\section{Apparatus}

The animal housing room was on a 12-h light/dark cycle. Lights were turned on at 7:00 a.m. and turned off at 7:00 p.m. The monkeys were housed in individual cages measuring $64 \mathrm{~cm} \times 72 \mathrm{~cm} \times$ $85 \mathrm{~cm}$ high. The cages were attached as a unit that was two cages high and two cages wide. A fluid-delivery panel, similar to that used in other studies (Meisch et al. 1975; Williams et al. 1998), was attached to one wall of each cage during daily sessions. Holes were cut in the cage wall so that two brass spouts on the fluid-delivery panel protruded into the cage $50 \mathrm{~cm}$ from the floor. A stimulus light that could be illuminated red or green was located $3 \mathrm{~cm}$ above each spout. The drinking solutions were contained in $1000 \mathrm{ml}$ plastic bottles attached to the back of the panel. Plastic tubing connected each bottle to the spout valve. The fluid containers were elevated so that the liquid was gravity-fed to the spout valve and delivery was controlled by a solenoid switch. Contact with either spout closed an electrical circuit (drinkometer) and a response was recorded. The stimulus light above the spout flashed when contact was made with the spout. When the reinforcement schedule was completed, the solenoid was activated and $0.5 \mathrm{ml}$ fluid was delivered. Solutions were measured after the session using graduated cylinders to confirm delivery amounts and check for gross discrepancies between the deliveries calculated by the computers and the volume gone from the bottles. The experiments were controlled and the data recorded using IBM PCjr microcomputers located in a room adjacent to the housing room. The same apparatus was used for the sucrose and PCP experiments.

\section{Procedure}

Each experimental session lasted $3 \mathrm{~h}$ per day, during which the animal could respond and obtain either ethanol or concurrently available water. Drug (ethanol) was available under the red stimulus light, and water was available under the green stimulus light. The side positions of the solutions were alternated daily. The monkeys were reinforced with $0.5 \mathrm{ml}$ fluid for every four mouth contacts on the spout. Thus, the reinforcement schedule was a fixed ratio 4 or FR4 schedule. The reinforcement schedule on each of the two spouts operated concurrently and independently such that the responses on one spout did not alter the number of responses required on the opposite spout. The experimental sessions were conducted at different times of the day depending upon the cages in which the monkeys were housed. For example, monkeys in the lower cages were in the first session which started at 7:00 a.m., and the monkeys in the upper cages were in the second session which started at 11:30 a.m. Just prior to the first session, the water hoses were disconnected from the cages. After the second session was finished the water hoses were reconnected and the monkeys were fed their daily ration of chow all at once. Sessions were conducted 7 days a week. These same procedures were used for the sucrose and PCP experiments.

The ethanol concentrations were $1 \%$ for two monkeys and $2 \%$ for the other three monkeys. These concentrations were previously shown to maintain the greatest amount of behavior (Williams et al. 1998). Due to the similarity, these data were pooled to calculate the average fluid deliveries and ethanol intake in $\mathrm{g} / \mathrm{kg}$. 
Saline or quadazocine $(0.32,1$, or $3.2 \mathrm{mg} / \mathrm{kg})$ injections were administered $30 \mathrm{~min}$ prior to the drinking sessions. This same pretreatment time was used in experiments 2 and 3. Each quadazocine dose was tested twice in each monkey in an ascending dose order. Some studies show enhanced sensitivity to the effects of opioid antagonists after repeated intermittent antagonist treatments (Warren and Morse 1985). Therefore, each quadazocine injection was given at least a week apart so that enhanced sensitivity did not develop in these monkeys. In addition 2-4 saline pretreatment days preceded the first antagonist injection day and were interspersed between other antagonist injection days. Non-injection days prior to the first antagonist injection day and between other antagonist or saline injection days were considered "baseline" days. These days served as a control for the saline injection days. Due to solubility problems, the largest quadazocine dose was given in three 1-ml syringes. This injection regimen was applied to the sucrose and PCP studies as well.

Experiment 2: sucrose self-administration

Subjects

Subjects were three male and two female monkeys. Two of the five subjects also participated in experiment 1 and three subjects received repeated NTX prior to sucrose access in an earlier experiment.

\section{Procedure}

Sucrose concentration $100 \mathrm{~g} / \mathrm{l}$ was previously determined to maintain a high amount of responding. Quadazocine doses $(0.032,1$, or $3.2 \mathrm{mg} / \mathrm{kg}$ ) were tested twice in each monkey in an ascending dose order for three monkeys and descending dose order for two monkeys.

\section{Experiment 3: PCP self-administration}

\section{Subjects}

Subjects were one female and four male monkeys. Two of the five subjects also participated in experiment 1 while three of the subjects received repeated NTX prior to sucrose access in an earlier experiment.

\section{Procedure}

Previously, we observed that the monkeys received most of their fluid deliveries during the first $40 \mathrm{~min}$ of the session (Williams et al. 1998). Additionally, we determined that the fluid deliveries obtained during a 3-h session within subjects were similar to those obtained during a 2-h session. Therefore, 2-h sessions were used for the PCP experiments to save time. Three of the five subjects in the present experiment self-administered PCP without induction procedures. The other two monkeys required minimal induction procedures. For example, during the first five sessions of PCP access, the monkeys received only $0.125 \mathrm{mg} / \mathrm{ml}$ PCP. No water was available during the session and the monkeys were fed after the session. Thereafter, water was introduced as the alternative solution. A PCP preference developed immediately. PCP sessions were conducted for approximately 2 weeks to establish a stable baseline. The best compromise between high rates of responding and drug intake $(\mathrm{mg} / \mathrm{kg})$ was at $0.125 \mathrm{mg} / \mathrm{ml}$ PCP. This PCP concentration was self-administered by monkeys in other studies (Carroll and Stotz 1984; Campbell et al. 1998). In each monkey, $1 \mathrm{mg} / \mathrm{kg}$ quadazocine was tested 4 times and $3.2 \mathrm{mg} / \mathrm{kg}$ quadazocine was tested twice. All four tests with $1 \mathrm{mg} / \mathrm{kg}$ were completed prior to testing with $3.2 \mathrm{mg} / \mathrm{kg}$. Both NTX doses, $0.1 \mathrm{mg} / \mathrm{kg}$ and $0.32 \mathrm{mg} / \mathrm{kg}$, were tested 4 times in each monkey. All four tests with $0.1 \mathrm{mg} / \mathrm{kg}$ were completed prior to testing with $0.32 \mathrm{mg} / \mathrm{kg}$.
Data analysis

Each monkey's average fluid deliveries, intake in $\mathrm{g} / \mathrm{kg}$, and fluid deliveries expressed as a percentage of non-injection baseline were used to calculate the mean and SE of the mean for the group of monkeys. The data are presented as the mean and SE of the mean for the group data.

The fluid delivery data for each antagonist pretreatment/solution condition (i.e. quadazocine/ethanol, quadazocine/sucrose, etc.) were analyzed separately using two-way repeated measures analysis of variance (RM ANOVA). A one-way RM ANOVA was used to analyze the drug intake data $(\mathrm{g} / \mathrm{kg}$ for ethanol or $\mathrm{mg} / \mathrm{kg}$ for PCP). For the ethanol, sucrose, or PCP data expressed as a percentage of non-injection baseline control, a one-way RM ANOVA was applied for each antagonist pretreatment/solution condition. The water data were not included in this analysis because the water baselines were low and small absolute changes in water fluid deliveries appeared large when expressed as a percent of non-injection baseline. The NTX/ethanol and NTX/sucrose data were taken from a previous study (Williams et al. 1998) and were presented here for comparison to quadazocine. When either the main effect of antagonist or the interaction effect of solution and antagonist was significant, post-hoc Dunnett's tests were used to compare the antagonist effects to saline control.

Drugs

Solutions were prepared by mixing the appropriate amounts of $95 \% \mathrm{w} / \mathrm{v}$ ethanol, sucrose (cane sugar), or PCP with tap water. Both NTX, provided by NIDA Research Technology Branch, and quadazocine, provided by Sterling-Winthrop, Collegeville, Pa., USA, were dissolved in sterile water.

\section{Results}

Experiment 1: ethanol self-administration

All quadazocine doses decreased ethanol fluid deliveries when compared to the ethanol fluid deliveries after saline injection as shown in Fig. 1 [interaction effect, $F(4,16)=10.76, P<0.001$; Dunnett's test $q^{\prime}=7.56,7.27$, and $6.66, P<0.05]$. Water fluid deliveries decreased after

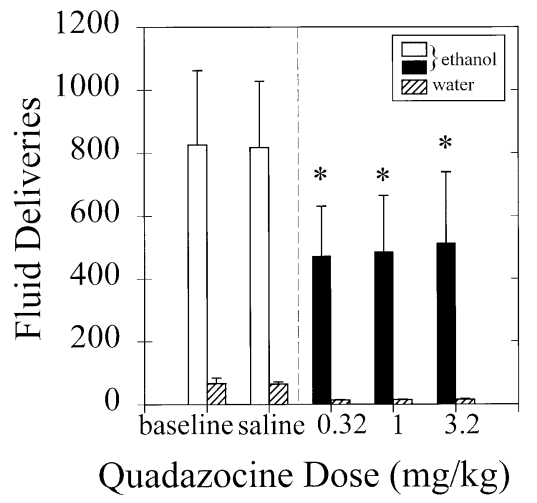

Fig. 1 The average number of fluid deliveries of ethanol (open and solid bars) and concurrently available water (lined bars) after different pretreatments: non-injection baseline, saline, quadazocine doses: $0.32,1$, and $3.2 \mathrm{mg} / \mathrm{kg}$. The bars represent the average with the SE ( $n=5$ monkeys; two consuming $1 \%$ ethanol and three consuming $2 \%$ ethanol). *Indicates a significant difference $(P<0.05)$ from ethanol fluid deliveries after saline 


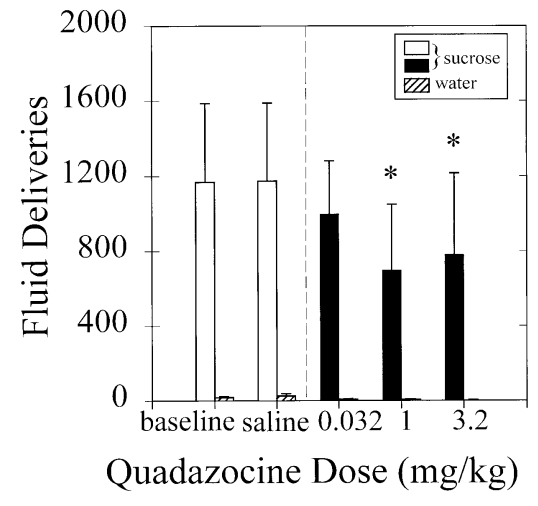

Fig. 2 The average number of fluid deliveries of $100 \mathrm{~g} / \mathrm{l}$ sucrose (open and solid bars) and concurrently available water (lined bars) after different pretreatments: non-injection baseline, saline, quadazocine doses: $0.032,1$, and $3.2 \mathrm{mg} / \mathrm{kg}$. The bars represent the average with the $\mathrm{SE}$ ( $n=5$ monkeys). *Indicates a significant difference $(P<0.05)$ from sucrose deliveries after saline

quadazocine, but this effect was not significant. Between monkeys, the average ethanol fluid deliveries varied from 394 to 1487 . Over the 3-h session, the average ethanol intake during baseline was $0.71 \pm 0.21 \mathrm{~g} / \mathrm{kg}$ and the average ethanol intake after saline was $0.70 \pm 0.18 \mathrm{~g} / \mathrm{kg}$. We did not observe behavioral signs of intoxication at these intake levels. All quadazocine doses reduced ethanol intake to approximately $0.4 \mathrm{~g} / \mathrm{kg}$ which was significantly different from the intake after saline $[F(4,16)=15$, $P<0.001$; Dunnett's test $q^{\prime}=4.85,5.05$, and 4.75, $P<0.05]$.

Experiment 2: sucrose self-administration

Quadazocine, 1 and $3.2 \mathrm{mg} / \mathrm{kg}$, reduced sucrose fluid deliveries when compared to the sucrose fluid deliveries after saline injection as shown in Fig. 2 [interaction effect, $F(4,16)=3.92, P<0.05$; Dunnett's test $q^{\prime}=4.45$ and 3.66, $P<0.05]$. The reduction in sucrose fluid deliveries after $1 \mathrm{mg} / \mathrm{kg}$ quadazocine was similar to that after $3.2 \mathrm{mg} / \mathrm{kg}$ quadazocine. The water fluid deliveries were almost zero under all conditions. Although the average data do not suggest a dose-related effect, quadazocine dose-dependently reduced sucrose fluid deliveries in three of the five monkeys. After $0.032 \mathrm{mg} / \mathrm{kg}$ quadazocine, which was 10 times less than the lowest dose tested on ethanol drinking monkeys, sucrose fluid deliveries remained similar to those during baseline and after saline.

\section{Experiment 3: PCP self-administration}

When PCP was available concurrently with water, the monkeys consumed $4.54 \pm 1.2 \mathrm{mg} / \mathrm{kg}$ PCP at baseline and $5.22 \pm 1.41 \mathrm{mg} / \mathrm{kg}$ after saline pretreatment. These intake levels produced behavioral effects of intoxication such

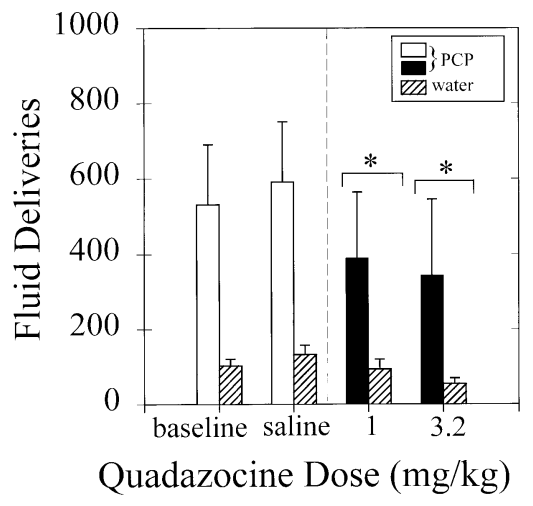

Fig. 3 The average number of fluid deliveries of $0.125 \mathrm{mg} / \mathrm{ml}$ phencyclidine (open and solid bars) and concurrently available water (lined bars) after different pretreatments: non-injection baseline, saline, quadazocine $1 \mathrm{mg} / \mathrm{kg}$, and quadazocine 3.2 $\mathrm{mg} / \mathrm{kg}$. The bars represent the average with the SE ( $n=5$ monkeys). *Indicates total fluid deliveries (PCP and water) significantly different $(P<0.05)$ from total fluid deliveries after saline

as ataxia and altered aggression in some monkeys. The concurrently available water maintained more fluid deliveries than during the ethanol or sucrose experiments. When quadazocine was given prior to PCP access, shown in Fig. 3, the interaction effect of quadazocine and solution was not significant $(P=0.056)$. Therefore, post-hoc comparisons were conducted for the main effect of quadazocine, which was significant $[F(3,12)=4.82$, $P<0.05]$. The results indicated that the total fluid deliveries of both PCP and water were reduced compared to the total fluid deliveries after saline injection [Dunnett's test $q^{\prime}=2.75$ and 3.38, $\left.P<0.05\right]$. Thus, neither PCP nor water was selectively reduced. Although $1 \mathrm{mg} / \mathrm{kg}$ quadazocine decreased PCP intake to $3.84 \pm 1.16 \mathrm{mg} / \mathrm{kg}$ and $3.2 \mathrm{mg} / \mathrm{kg}$ quadazocine decreased PCP intake to $2.78 \pm 1.50 \mathrm{mg} / \mathrm{kg}$, these intakes were not significantly different from the PCP intake after saline $(P=0.11)$.

Both NTX doses, 0.1 and $0.32 \mathrm{mg} / \mathrm{kg}$, reduced PCP fluid deliveries [interaction effect, $F(3,12)=4.89$, $P<0.05$; Dunnett's test $q^{\prime}=4.48$ and 5.05, $\left.P<0.05\right]$ without affecting water fluid deliveries, as shown in Fig. 4. The monkeys consumed $5.62 \pm 0.9 \mathrm{mg} / \mathrm{kg}$ PCP at baseline, $\quad 5.37 \pm 1.31 \mathrm{mg} / \mathrm{kg}$ after saline pretreatment, $2.71 \pm 0.62 \mathrm{mg} / \mathrm{kg}$ after $0.1 \mathrm{mg} / \mathrm{kg}$ NTX, and $2.51 \pm$ $0.62 \mathrm{mg} / \mathrm{kg}$ after $0.32 \mathrm{mg} / \mathrm{kg}$ NTX. Both NTX doses significantly reduced PCP intake when compared to PCP intake after saline injection $[F(3,12)=11.8, P<0.0001$; Dunnett's test $q^{\prime}=3.86$ and $\left.4.16, P<0.05\right]$.

The effects of quadazocine and NTX on the fluid deliveries of ethanol, sucrose, and PCP are expressed as percentage of non-injection baseline control in Fig. 5. Because some subjects were used in multiple antagonist/solution conditions and others were not, we could only make statistical comparisons within each antagonist pretreatment/solution condition. Quadazocine, 1 and $3.2 \mathrm{mg} / \mathrm{kg}$, reduced ethanol $[F(3,12)=9.52, P<0.01$; Dunnett's test $q=4.32$ and 4.62, $P<0.05], \quad$ sucrose $\left[F(3,12)=6.23, P<0.01 ;\right.$ Dunnett's test $q^{\prime}=3.36$ and 3.41, 


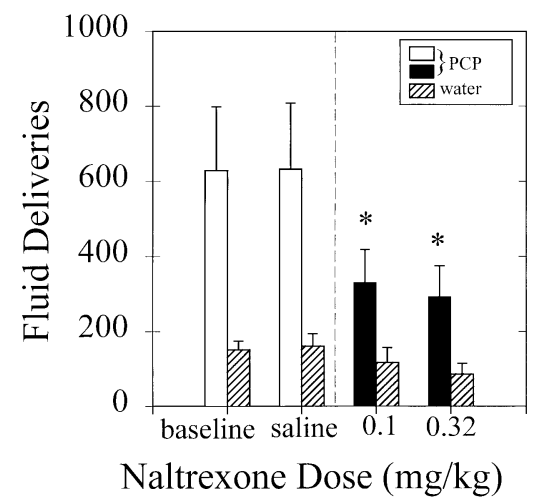

Fig. 4 The average number of fluid deliveries of $0.125 \mathrm{mg} / \mathrm{ml}$ phencyclidine (open and solid bars) and concurrently available water (lined bars) after different pretreatments: non-injection baseline, saline, naltrexone $0.1 \mathrm{mg} / \mathrm{kg}$, and naltrexone $0.32 \mathrm{mg} / \mathrm{kg}$. The bars represent the average with the SE ( $n=5$ monkeys). *Indicates a significant difference $(P<0.05)$ from phencyclidine fluid deliveries after saline

$P<0.05]$, and PCP $[F(2,8)=6.48, P<0.05$; Dunnett's test $q^{\prime}=2.64$ and 3.44, $\left.P<0.05\right]$ to a similar extent. Although $0.032 \mathrm{mg} / \mathrm{kg}$ quadazocine was ineffective when tested against sucrose, $0.32 \mathrm{mg} / \mathrm{kg}$ quadazocine reduced ethanol to the same degree that 1 and $3.2 \mathrm{mg} / \mathrm{kg}$ quadazocine reduced ethanol. NTX, $0.32 \mathrm{mg} / \mathrm{kg}$, reduced ethanol $\left[F(3,12)=6.47, P<0.01\right.$; Dunnett's test $\left.q^{\prime}=4.19, P<0.05\right]$, sucrose $\left[F(2,8)=32.7, P<0.001\right.$; Dunnett's test $q^{\prime}=8.04$, $P<0.05]$, and PCP $[F(2,8)=10.2, P<0.01$; Dunnett's test $\left.q^{\prime}=4.31, P<0.05\right]$. NTX, $0.1 \mathrm{mg} / \mathrm{kg}$, reduced only sucrose and PCP [Dunnett's test $q^{\prime}=3.24$ and 3.37, $P<0.05]$. With all solutions, NTX appeared to produce dose-related effects. Overall, the quadazocine-induced reductions were not as dose-related as the NTX-induced reductions.

\section{Discussion}

The results showed that quadazocine reduced ethanol and sucrose fluid deliveries without affecting the concurrently available water. Although quadazocine failed to selectively reduce PCP fluid deliveries, Fig. 5 indicates that the significant reduction of total fluid deliveries (PCP and water) was primarily due to reduced PCP fluid deliveries. In addition, NTX reduced PCP fluid deliveries without affecting water. When the antagonist effects were compared across all solutions as a percentage of non-injection baseline control, the effects of NTX and quadazocine were similar.

The endogenous opioid system may modulate ethanol-, sucrose-, and PCP-reinforced responding. Some researchers postulate that increased opioid activity modulates ethanol reward and that NTX may reduce ethanol drinking by blocking the reward associated with the ethanol-induced opioid activity (Volpicelli et al. 1992; Gianoulakis and de Waele 1994). If NTX blocks the endogenous opioids released by ethanol, then increasing ethanol dose should overcome the effects of the opioid antagonist. However, our data do not fully support this hypothesis. In a previous paper (Williams et al. 1998), we showed that the NTX effect on oral and intravenous ethanol self-administration was not surmountable by increasing the available ethanol concentration or dose. In addition, the present study shows that the opioid antagonist quadazocine reduced ethanol-, sucrose-, and PCP-reinforced responding. Furthermore, NTX doses that reduced ethanol-reinforced responding also reduced sucrose- and PCP-reinforced responding. If the ethanolopioid hypothesis is correct, then our data suggest that ethanol, sucrose, and PCP must increase opioid activity to the same degree. If the endogenous opioid activity af-
Fig. 5 The average number of fluid deliveries of ethanol (left panels), sucrose (middle panels), and phencyclidine (right panels), expressed as a percentage of non-injection baseline control after saline or quadazocine pretreatment (upper panels) and after saline or naltrexone pretreatment (lower panels). *Indicates a significant difference $(P<0.05)$ from the saline for that particular antagonist/solution condition

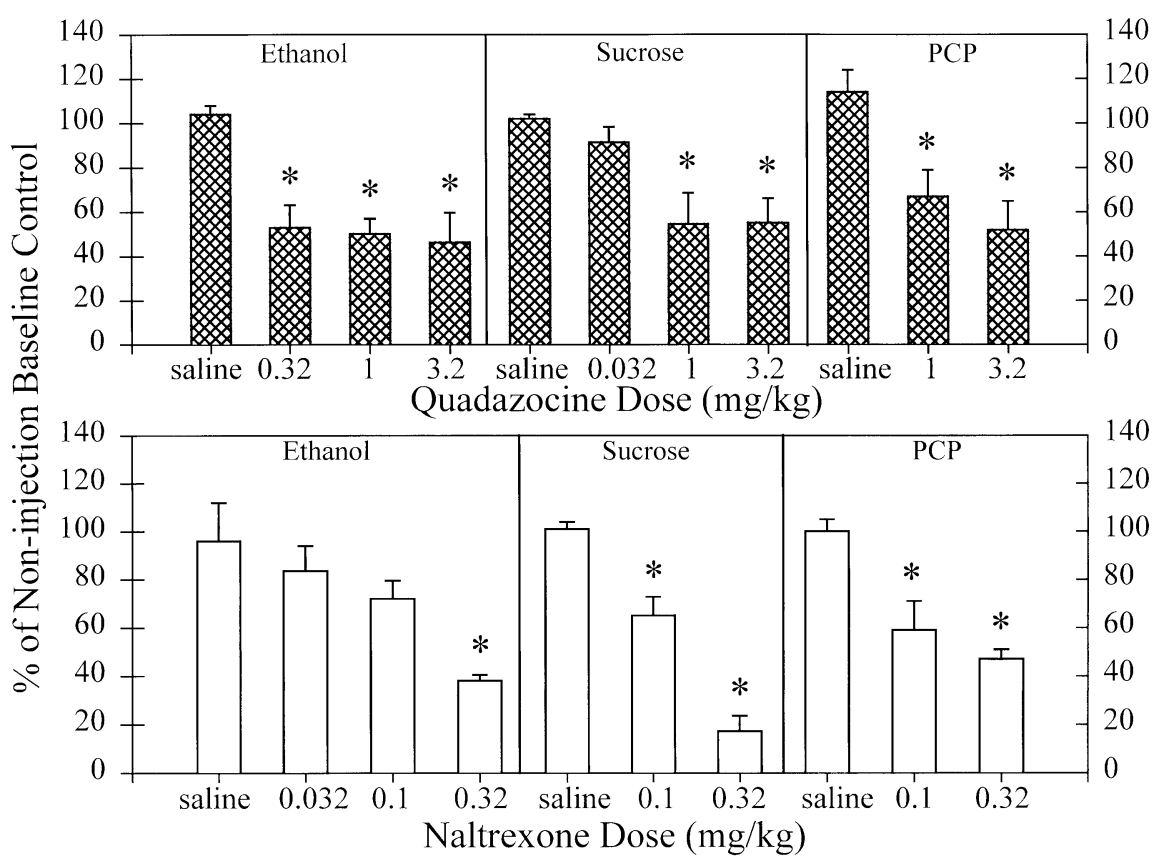


ter drinking sucrose or PCP was less than that after drinking ethanol, we might expect much smaller quadazocine or NTX doses to reduce sucrose- and PCP-reinforced responding.

Opioid receptors appear to mediate the antagonist-induced reduction in oral-reinforced responding. Although the opioid antagonist doses used were quite large, our data indicate that the antagonist interaction with opioid receptors mediates the reduction of oral-reinforced responding. Earlier studies showed that a 10-fold higher quadazocine dose was needed to precipitate withdrawal or generalize to a NTX stimulus in morphine-dependent monkeys (Valentino et al. 1983; France et al. 1990). Since morphine acts primarily through $\mu$-receptors, we postulated that if NTX reduces oral-reinforced responding through the $\mu$-receptor, then quadazocine would produce similar effects at a 10-fold higher dose than NTX. In our study, fluid deliveries were reduced after 1-3.2 $\mathrm{mg} / \mathrm{kg}$ quadazocine and $0.1-0.32 \mathrm{mg} / \mathrm{kg}$ NTX. These results support our hypothesis. Because the potency of quadazocine relative to NTX matches that previously studied with morphine withdrawal, we conclude that opioid receptors, probably of the $\mu$-subtype, mediate antagonist-induced reductions in oral-reinforced responding. This conclusion differs from that in a previous study (Williams and Woods 1998a). In that study, we concluded that the $\mu$-receptor did not mediate the antagonist-induced reduction in oral-reinforced responding because an irreversible $\mu$-antagonist that was highly effective in other assays failed to affect ethanol-reinforced responding. However, antagonist availability and solubility issues prevented us from testing higher doses.

Enhanced sensitivity to opioid antagonists may be a confounding factor in our experiments. In previous experiments with squirrel monkeys responding for food, repeated NTX administration enhanced the monkeys' sensitivity to the rate-decreasing effects of NTX (Warren and Morse 1985). In addition, rats with enhanced NTX sensitivity showed cross-sensitivity to other opioid antagonists (Schindler et al. 1993). This enhanced sensitivity is observed in animals responding for food, but not in animals responding to avoid a shock (France and Morse 1989; Warren and Morse 1989). Animals may develop enhanced sensitivity to NTX because the NTX-induced interoceptive state may become conditionally associated with the food or taste stimulus over repeated testing. We tried to avoid this sensitivity issue in our monkeys by pretreating with antagonists only once per week and giving many saline injections between antagonist pretreatments. In spite of our precautions, enhanced sensitivity or conditioned association may account for the reduced ethanol-reinforced responding after $0.32 \mathrm{mg} / \mathrm{kg}$ quadazocine. In the quadazocine/ethanol experiment, two of the five monkeys had previously been exposed to repeated intermittent injections of NTX prior to ethanol or sucrose access. Because many of these animals received repeated NTX injections prior to ever receiving quadazocine, enhanced sensitivity may have contributed to quadazocine's effect on reinforced responding.
The present experiments also have some other limitations. The quadazocine dose-range was limited and smaller quadazocine doses should have been used. Because smaller doses were not tested and the dose-effect curves appear flat, we simply do not know if smaller quadazocine doses would decrease oral-reinforced responding. Another potential limitation is that quadazocine and NTX were tested at ethanol and sucrose concentrations where responding for the concurrently available water was low. This problem raises the question of whether the antagonist effects were rate-dependent. In preliminary experiments (Williams and Woods 1998b), we examined the effect of NTX at ethanol concentrations where ethanol maintained more, equal to, or less fluid deliveries than the concurrently available water. NTX reduced the fluid deliveries of the fluid that maintained the greatest behavior whether it was ethanol or water. Although opioid antagonists reduce responding reinforced by ethanol, sucrose, and PCP, it is difficult to determine how the antagonists produce these effects. Some research suggests that opioid antagonists reduce palatability (Cooper and Turkish 1989; Giraudo et al. 1993). In the present study, ethanol and sucrose may be palatable while the PCP has taste qualities similar to quinine (Aspen 1997). The fact that PCP fluid deliveries were reduced by antagonist pretreatment suggests that the palatability explanation fails to explain fully the antagonist effects. In addition, because PCP has no caloric content, the antagonist effects are probably not mediated by a mechanism governing caloric intake. Also, the antagonist effects are unlikely to be related to the pharmacological properties of the solutions because responding for the non-drug sucrose solution was reduced. Another alternative is that opioid antagonists produce nausea or aversive effects that reduce consummatory behaviors. Opioid antagonists have been shown to produce conditioned aversions in rodents (Mucha and Walker 1987; Parker and Rennie 1992) and at least one clinical trial with alcohol-dependent subjects reported nausea as a side-effect of NTX (O'Malley 1992).

Although this paper suggests that opioid receptors (perhaps $\mu$-receptors) mediate the effects of opioid antagonists on oral-reinforced behaviors, some points remain to be clarified. For example, much larger antagonist doses are required to reduce consumption than to block an exogenous opioid effect such as analgesia. At these larger doses, NTX and quadazocine block $\mu-, \kappa-$, and $\delta$-opioid receptors (Negus et al. 1993; Ko et al. 1998). The necessary use of large antagonist doses to reduce oral-reinforced responding may indicate that opioid receptors are not the sole mediators of the antagonist effects on reinforced responding. In addition, it is not understood why the NTX effect on oral- and intravenousreinforced responding is not surmountable by increasing the ethanol concentration or dose (Williams et al. 1998). Also, the role of enhanced sensitivity and conditioning in the antagonist effect on oral-reinforced responding remains unexplained. It is not clear how these factors interact or whether they have significance in clinically treating alcoholism with opioid antagonists. 
Acknowledgements This work was supported by USPHS grants AA-11424 and DA08568.

\section{References}

Aspen JM (1997) Taste discrimination of opioid and phencyclidine-like drugs in rhesus monkeys. Unpublished $\mathrm{PhD}$ thesis, University of Chicago, Chicago

Brown DR, Holtzman SG (1980) Evidence that opiate receptors mediate suppression of hypertonic saline-induced drinking in the mouse by narcotic antagonists. Life Sci 26:1543-1550

Campbell UC, Thompson SS, Carroll ME (1998) Acquisition of oral phencyclidine (PCP) self-administration in rhesus monkeys: effects of dose and alternative non-drug reinforcer. Psychopharmacology 137:132-138

Carroll ME (1982) Rapid acquisition of oral phencyclidine selfadministration in food-deprived and food-satiated rhesus monkeys: concurrent phencyclidine and water choice. Pharmacol Biochem Behav 17:341-346

Carroll ME, Stotz DC (1984) Increased phencyclidine self-administration due to food deprivation: interaction with concentration and training conditions. Psychopharmacology 84:299-303

Cooper SJ, Turkish S (1989) Effects of naltrexone on food preference and concurrent behavioral responses in food-deprived rats. Pharmacol Biochem Behav 33:17-20

De Witte P (1984) Naloxone reduces alcohol intake in a freechoice procedure even when both drinking bottles contain saccharin sodium or quinine substances. Biol Psychiatry 12:7377

Emmerson PJ, Liu M-R, Woods JH, Medzihradsky F (1994) Binding affinity and selectivity of opioids at $m u$, delta, and kappa receptors in monkey brain membranes. J Pharmacol Exp Ther 271:1630-1637

France CP, Morse WH (1989) Pharmacological characterization of supersensitivity to naltrexone in squirrel monkeys. J Pharmacol Exp Ther 250:928-936

France CP, De Costa BR, Jacobson AF, Rice KC, Woods JH (1990) Apparent affinity of opioid antagonists in morphinetreated rhesus monkeys discriminating between saline and naltrexone. J Pharmacol Exp Ther 252:600-604

Froehlich JC, Harts J, Lumeng L, Li T-K (1990) Naloxone attenuates voluntary ethanol intake in rats selectively bred for high ethanol preference. Pharmacol Biochem Behav 35:385-390

Gianoulakis C, de Waele JP (1994) Genetics of alcoholism: role of endogenous opioid system. Metab Brain Dis 9:105-131

Giruado SQ, Grace MK, Welch CC, Billington CJ, Levine AS (1993) Naloxone's anorectic effect is dependent upon the relative palatability of food. Pharmacol Biochem Behav 46:917921

Holtzman SG (1974) Behavioral effects of separate and combined administration of naloxone and $d$-amphetamine. J Pharmacol Exp Ther 189:51-60

Kirkham TC, Cooper SJ (1988) Attenuation of sham feeding by naloxone is stereospecific: evidence for opioid mediation of orosensory reward. Physiol Behav 43:845-847

Ko M-C, Butelman ER, Traynor JR, Woods JH (1998) Differentiation of kappa opioid agonist-induced antinociception by nal- trexone apparent $\mathrm{pA}_{2}$ analysis in rhesus monkeys. J Pharmacol Exp Ther 285:518-526

Levine AS, Billington CJ (1989) Opioids: are they regulators of feeding. Ann NY Acad Sci 575:209-219

Levine AS, Morley JE, Gosnell BA, Billington CJ, Bartness TJ (1985) Opioids and consummatory behavior. Brain Res Bull 14:663-672

Maickel RP, Braude MC, Zabik JE (1977) The effects of various narcotic agonists and antagonists on deprivation-induced fluid consumption. Psychopharmacology 16:863-866

Mason BJ, Ritvo EC, Morgan RO, Salvato FR, Goldberg G, Welch B, Mantero-Atienza E (1994) A double-blind, placebocontrolled pilot study to evaluate the efficacy and safety of oral nalmefene $\mathrm{HCl}$ for alcohol dependence. Alcohol Clin Exp Res 18:1162-1167

Meisch RA, Henningfield J, Thompson T (1975) Establishment of ethanol as a reinforcer for rhesus monkeys via the oral route: initial results. Adv Exp Med Biol 59:323-342

Mucha RF, Walker MJK (1987) Aversive property of opioid receptor blockade in drug-naive mice. Psychopharmacology 93: 483-488

Negus SS, Burke TF, Medzihradsky F, Woods JH (1993) Effects of opioid agonists selective for mu, kappa, and delta opioid receptors on schedule-controlled responding in rhesus monkeys: antagonism by quadazocine. J Pharmacol Exp Ther 267:896903

O'Malley SS, Jaffe AJ, Chang G, Shottenfeld R, Meyer RE, Rounsaville B (1992) Naltrexone and coping skills therapy for alcohol dependence. Arch Gen Psychiatry 49:881-887

Parker LA, Rennie M (1992) Naltrexone-induced aversions: assessment by place conditioning, taste reactivity, and taste avoidance paradigms. Pharmacol Biochem Behav 41:559-565

Schindler CW, Goldberg SR, Katz JL (1993) Pharmacological specificity of enhanced sensitivity to naltrexone in rats. Psychopharmacology 110:60-68

Stapleton JM, Ostrowski NL, Merriman VJ, Lind MD, Reid LD (1979) Naloxone reduces fluid consumption in water-deprived and non-deprived rats. Bull Psychon Soc 13:237-239

Ulm RR, Volpicelli JR, Volpicelli LA (1995) Opiates and alcohol self-administration in animals. J Clin Psychiatry 56:5-14

Valentino RJ, Katz JL, Medzihradsky F, Woods JH (1983) Receptor binding, antagonist, and withdrawal, precipitating properties of opiate antagonists. Life Sci 32:2887-2896

Volpicelli JR, Alterman AI, Hayashida M, O'Brien CP (1992) Naltrexone in treatment of alcohol dependence. Arch Gen Psychiatry 49:876-880

Warren PH, Morse WH (1989) Environmental determinants of enhanced sensitivity to the behavioral effects of naltrexone. J Pharmacol Exp Ther 248:546-551

Williams KL, Winger G, Pakarinen ED, Woods JH (1998) Naltrexone reduces ethanol- and sucrose-reinforced responding in rhesus monkeys. Psychopharmacology 139:53-61

Williams KL, Woods JH (1998a) Oral ethanol-reinforced responding in rhesus monkeys: effects of opioid antagonists selective for the mu-, kappa-, or delta-receptor. Alcohol Clin Exp Res 22:1634-1639

Williams KL, Woods JH (1998b) Insurmountable antagonist and ethanol concentration dependent effects of naltrexone on ethanol drinking in rhesus monkeys. Alcohol Clin Exp Res 22:14A 